\title{
RESEARCH / INVESTIGACIÓN \\ La gestión ambiental en una entidad hotelera de turismo de naturaleza. Caso Villa Mirador de Mayabe de Holgín, Cuba
}

\section{Environmental management in a nature tourism hotel. The case of Villa Mirador de Mayabe de Holguín, Cuba}

Ana María Azze Alonso¹, Migdely Barbarita Ochoa Ávila²

Resumen: Esta investigación aborda la gestión ambiental en la Villa Mirador de Mayabe perteneciente a la empresa Islazul Holguín, la cual está ubicada en las alturas de Mayabe a 12 km del centro histórico de Holguín en el oriente de Cuba. El objetivo fue aplicar un procedimiento general para la gestión ambiental que contribuya a la mejora del medio ambiente. Dicho procedimiento permitió desplegar una estrategia para mejorar su desempeño ambiental, lo cual es un requisito fundamental para la implementación de los procesos de perfeccionamiento empresarial y categorización hotelera por parte del Ministerio del Turismo. Se abordan los conceptos y reflexiones sobre medio ambiente y su interrelación e importancia para la actividad turística, que fundamentan la aplicación del procedimiento el que permitió diagnosticar, planificar, establecery dar seguimiento a la gestión ambiental en la Villa en el período 2007-2015. El procedimiento general para la gestión ambiental permitió a la instalación hotelera formular e implementar estrategias efectivas para mejorar el medio ambiente y el uso responsable de recursos, adaptándose a los cambios del micro y macro entorno. Además, logró cambios favorables en la generalidad de los indicadores aplicados para su evaluación.

Palabras clave: Gestión ambiental, procedimiento general, uso responsable de recursos.
Abstract: This study addresses the environmental management in the Villa Mirador de Mayabe, which belongs to the company Islazul Holguin, located atop Mayabe $12 \mathrm{~km}$ from the historical center of Holguin in eastern Cuba. The aim was to apply a general procedure for environmental management that contributes to the improvement of the environment. With this procedure, a strategy was presented to improve its environmental performance, which is a fundamental requirement for the implementation of the managerial improvement and hotel rating processes by the Ministry of Tourism. The concepts and reflections on the environment and its interrelation and importance for tourist activity are addressed, as these form the foundation of the application of the procedure that permitted the diagnosis, planning, establishment and follow-up to the environmental management at the Villa in the period 2007-2015. The general procedure for environmental management enabled the hotel to formulate and implement effective strategies to improve the environment and the responsible use of resources, adapting to micro and macroenvironmental changes. In addition, favorable changes were made in most of the indicators applied for its assessment.

Keywords: Environmental management, general procedure, responsible use of resources.

(Presentado: 09 de mayo de 2016. Aceptado: 4 de junio de 2016)

\footnotetext{
${ }^{1}$ Especialista en Gestión Hotelera. Especialista de la Empresa Turística de Gibara, Delegación Territorial del Ministerio del Turismo de Holguín, Cuba E-mail: daimara.reyes Qcubanacanhlg.tur.cu

${ }^{2}$ Doctora en Ciencias Técnicas. Facultad de Ingeniería Industrial y Turismo, Universidad de Holguín, Cuba. E-mail: migdelyafacii.uho.edu.cu
} 


\section{INTRODUCCIÓN}

El turismo es un servicio mundialmente expandido en la actualidad por los resortes económicos que mueve. Puede generar impactos perjudiciales si su crecimiento acelerado sólo se dirige a obtener beneficios económicos, si no se aplica un adecuado manejo de los recursos naturales, materiales e inmateriales que lo sustentan, y además si no se tienen en cuenta las leyes que rigen el equilibrio de los ecosistemas. Los mayores impactos de la actividad turística producen afectaciones a la diversidad biológica, fundamentalmente sobre los ecosistemas costeros, como las playas, lagunas, manglares, arrecifes coralinos y otros; así como en el orden social entre otros (Colectivo de autores, 2007). El turismo en Cuba es uno de los renglones que más ingresos económicos genera, así la cultura, historia y naturaleza son los fundamentales atractivos para los visitantes de diversas partes del mundo. La construcción de infraestructuras de hospedaje, servicios, el desarrollo de áreas recreativas, y todas las acciones que se realizan de forma directa o indirecta para prestar servicio a los visitantes, contribuyen al deterioro del medio ambiente, de ahí la necesidad de fomentar un turismo sostenible en todos los aspectos.

La modalidad turística de naturaleza tiene un gran crecimiento a nivel mundial. Se le ha conferido diferentes denominaciones, tales como turismo ecológico, ecoturismo, turismo verde o turismo de naturaleza. No obstante se debe tener presente que en este tipo de actividad turística, cualquiera sea su denominación, se requiere un uso más racional de los recursos naturales y la utilización de tecnologías más eficientes y menos contaminantes.

Esta investigación aborda la gestión ambiental en la Villa Mirador de Mayabe perteneciente a la empresa Islazul Holguín, la cual está ubicada en las alturas de Mayabe, a $12 \mathrm{~km}$ del centro histórico de Holguín en el oriente de Cuba. Es un lugar emblemático en esta región cubana, rodeada de una bella naturaleza y como atractivo el burro "Pancho", que toma cerveza, el que le ha dado fama. La Villa se distingue dentro de las ofertas turísticas de Holguín, la ciudad de los parques. Para efectos de este trabajo se considera que "Villa turística" es un conjunto de cabañas que permite a sus huéspedes, bajo condiciones previamente determinadas, disfrutar de sus vacaciones en contacto directo con la naturaleza (Oficina Nacional de Estadística de Cuba, 2015).
La Villa Mirador de Mayabe posee 24 habitaciones, de ellas 19 estándares, 1 suite y 4 en la "Casa Pancho", todas climatizadas, con televisión satelital, agua caliente, caja de seguridad, mini bar y teléfono. El servicio gastronómico se brinda en dos restaurantes a manera de ranchón, uno de ellos es la "Finca Mayabe", que de conjunto con la "Casa del Campesino" forman un producto genuinamente cubano. Cuenta además con snack-bar, servibar 24 horas, Parrillada "Los Caneyes" y la "Eco cava Mayabe". También se ofrecen servicios de piscina, atención médica, parqueo, masaje, tienda y "Tea Garden". Los principales mercados de la Villa son Canadá, Emigrados Cubanos, Reino Unido y España.

Desde 2013 la instalación opera bajo la marca Masnatura que a partir de la concepción de los hoteles Islazul está fundamentada en brindar una oferta superior, en hoteles ubicados en parques o zonas naturales de categoría 2 y 3 estrellas, con vistas a brindar un servicio especialmente para los recorridos especializados de los receptivos, para la pesca y actividades de turismo especializado, con una variada oferta gastronómica. El objetivo de esta diferenciación de marca radica en que en dichas instalaciones los clientes deben encontrar un ambiente cordial y un personal especializado en las actividades a desarrollar. Además se debe garantizar una ambientación natural, rústica, que dé sentido de integración entre lo humano y lo natural, que emplee los recursos autóctonos, el talento artístico y las más genuinas tradiciones de la localidad. A raíz de la implementación de un Sistema de Gestión y Dirección Empresarial en la empresa Islazul Holguín, la Villa comenzó a realizar acciones para incorporar la gestión ambiental a su sistema general, a partir de que se detectaron las siguientes insuficiencias:

- No contaba con un Sistema de Gestión Ambiental.

- Insuficiente manejo de recursos naturales y energéticos.

- Insuficiente conocimiento en la temática ambiental.

- Carecía de una estrategia ambiental para alcanzar de forma sistémica, participativa y por procesos los objetivos y metas ambientales que favorecieran un comportamiento consciente hacia la conservación de los valores que hacen tan atractiva esta instalación turística. 
Lo anterior implica que como problema científico se plantea que las insuficiencias en la gestión ambiental limitan la mejora del medio ambiente en la Villa Mirador de Mayabe. Para esta investigación se seleccionó como objeto de investigación la gestión ambiental en entidades hoteleras de turismo de naturaleza. Al tener en cuenta la necesidad de que en la Villa se tome conciencia de las exigencias que se plantean a nivel internacional y nacional en cuanto a la gestión ambiental en entidades hoteleras, se definió como objetivo general el siguiente: Aplicar un procedimiento general para la gestión ambiental en la Villa Mirador de Mayabe que contribuyera a la mejora del medio ambiente. Para dar cumplimiento al objetivo general resultó necesario dar consecución a los objetivos específicos siguientes:

- Elaborar los fundamentos teóricos-prácticos referenciales que sustentan la gestión ambiental en entidades hoteleras de turismo de naturaleza, y

- Aplicar un procedimiento general para la gestión ambiental en la Villa Mirador de Mayabe.

\section{La gestión ambiental en las instalaciones hoteleras enmarcadas en el turismo de naturaleza}

La gestión ambiental resulta de gran importancia para las instalaciones hoteleras en general a nivel de planeta. Son múltiples las definiciones de gestión ambiental aplicadas a organizaciones turísticas; en su mayoría están relacionadas con las acciones encaminadas a la protección y uso sostenible del medio ambiente. Para Atencio Zayas (2016) la gestión ambiental es un campo temático amplio que hace énfasis en la aplicación de mecanismos normativos y técnicas encaminadas a fortalecer la capacidad humana e institucional para abordar eficazmente las cuestiones ambientales. Se coincide con: "El desarrollo del turismo (...), afecta a la gestión ambiental de varias formas; en primer lugar por el tipo de actividades que realiza el cliente durante su estancia y su nivel de contacto y presencia en el establecimiento con el entorno natural; en segundo lugar, por la duración media de su hospedaje, que concede un mayor margen para emprender distintas iniciativas ambientales en función de dicha duración, finalmente por el motivo seleccionado por el cliente para elegir el establecimiento y en especial, la importancia que el cliente le otorga a los atractivos turísticos naturales (Ayuso, 2003, en González-Abreu, 2016). Se parte de los establecido por Salinas Chávez (2008) acerca del espacio turístico y su clasificación en dos aspectos importantes; las características de los paisajes existentes y los usos socio-económicos predominantes en cada uno de ellos, por lo que considera que se clasifican en cuatro tipos: litorales, naturales, rurales y urbanos. Además, deben reunir siempre un mínimo de condiciones y valores naturales en buen estado de conservación, los cuales son de interés para los visitantes. La modalidad turística que tiene como destino la naturaleza tiene un gran crecimiento a nivel mundial aunque se le da diferentes denominaciones, como turismo ecológico, ecoturismo, turismo verde o turismo de naturaleza. Lo que distingue este tipo de actividad turística es que requiere un uso más racional de los recursos naturales y la utilización de tecnologías más eficientes y menos contaminantes.

Los recursos naturales han constituido la primera base de motivación para los viajes turísticos. Ver, vivir y experimentar el escenario natural de cualquier región constituye hoy uno de los principales propósitos para desplazarse a un sitio determinado. De ahí la importancia de la conservación de los sistemas naturales y el uso racional de los recursos con el objetivo de evitar la destrucción del entorno. Una buena forma de tratar los atractivos turísticos de manera que sean rentables y conservados es al tomar conciencia de que los recursos turísticos son limitados. El impacto negativo de las actividades turísticas en el medio ambiente se puede apreciar fundamentalmente en la alteración de zonas naturalmente frágiles ocasionado por el desarrollo de infraestructura vial, deforestación, erosión de senderos de acceso a las áreas naturales, lanzamiento de desperdicios, contaminación de las aguas, entre otros. Además se produce un impacto negativo con consecuencias a largo plazo, y que corresponde a la excesiva carga ambiental sobre un área determinada. Esta sobrecarga incide significativamente en el ecosistema, afectando el comportamiento de los animales, en sus tasas de reproducción, e incluso en la composición de las diversas especies biológicas presentes en el lugar. Esto ocurre mayormente debido a la falta de metodologías adecuadas para evaluar el impacto del turismo en el medio ambiente y en sus componentes bióticos (Cruz-Rodríguez, 2010).

La construcción de infraestructuras de hospedaje, servicios, el desarrollo de áreas recreativas, y todas las acciones que se realizan de forma directa o indirecta para prestar servicio a los visitantes, contribuyen al deterioro del medio ambiente, de ahí la necesidad de fomentar un turismo sostenible en todos los aspectos, esto se hace más notorio cuando se trata de instalaciones hoteleras insertadas en un espacio turístico natural. La Organización Mundial de 
Turismo (OMT) junto a otras organizaciones tradicionales del sector, cada día más, promueven la sensibilidad y las demandas de los viajeros sobre la conservación del medio ambiente, lo que constituye un elemento de suma importancia para las zonas turísticas que se encuentran enclavadas en lugares naturales y cuyo entorno desempeña un rol esencial en su proyección como producto. La adopción de los principales parámetros que se han establecido a nivel mundial y la conservación responsable del medio ambiente, garantizarán una buena posición en el mercado y el éxito de ganar competitividad (Carmona-Moreno y Magán-Díaz, 2008; Marrero-Nápoles, 2010). El turismo de naturaleza en Cuba es un ejemplo concreto y práctico del alto grado de coordinación interinstitucional y de articulación funcional que propician la estructura social cubana, y el Estado, en aras de alcanzar propósitos concurrentes a variados organismos y generarle beneficios a la sociedad en su conjunto. La pródiga, variada y bien conservada naturaleza cubana es uno de los atractivos principales del turismo en el país (Polanco-Charchabal, 2014). La concepción de los hoteles Masnatura adoptada en 2012 por el Grupo Hotelero Islazul en Cuba está fundamentada en brindar una oferta superior, en hoteles ubicados en parques o zonas naturales de categoría 2 y 3 estrellas. Los hoteles son Hotel Hanabanilla, Villa San José del Lago, Villa Santo Domingo, Mirador de San Diego, Balcón de la Sierra, Gran Piedra, Hotel Pasacaballo, Villa Yaguanabo, y Villa Mirador de Mayabe.

En el Manual Operativo de la marca Masnatura se plantea que en estas instalaciones los clientes deben encontrar un ambiente cordial y que el personal debe ser especializado en las actividades a desarrollar. La marca establece estándares físicos y de servicio en estas instalaciones hoteleras enclavadas en espacios turísticos de naturaleza, para controlar su cumplimiento en correspondencia con las normativas del sector turístico en el país. Algunos de los principales rasgos distintivos de la marca Masnatura son los siguientes:

- Ornamentación con plantas o flores naturales;

- El eslogan de la marca es "Compartir la naturaleza" o "Cubanos por naturaleza";

- Rueda de menú para buffet, menú corporativo para Snack bar, bares, y restaurantes especializados en comida internacional, con algunos platos regionales;

- Tarjetas de llaves o porta llaves con el logo rústicos adaptados al entorno natural;
- Ceniceros de áreas rústicos adaptados al entorno natural;

- Señalización de plantas endémicas y de más antigüedad en la zona;

- Habitaciones para no fumadores (prescindiendo de ceniceros en ellas);

- Potenciar la siembra de árboles de conjunto trabajadores, directivos y clientes repitentes de las instalaciones;

- Potenciar el auto consumo y las formas productivas;

- Protección de la naturaleza y la preservación del medio ambiente;

- Por una gastronomía auténtica, variada y regional;

- Colocar bayas, gigantografía, carteles con mensajes alegóricos al cuidado del medio ambiente;

- Anuncios ecológicos en exteriores e interiores de las instalaciones y en baños de habitación;

- Eco cava (lugar donde el cliente pueda degustar el vino de la casa y otros de una manera diferente);

- Ceniceros de madera en forma de tronco;

- Balances, bancos, columpios, taburetes o hamacas rústicos;

- Carta menú con forro de pajilla como los de los balances y el menú escrito a mano (el papel utilizado también es el manufacturado); y

- Menú vegetariano.

Debido a su relevancia, esta investigación está centrada en la gestión ambiental en una instalación turística hotelera enmarcada en turismo de naturaleza, la Villa Mirador de Mayabe, la cual influye directamente en la oferta de un producto con excelencia y al cumplimiento de los estándares de la marca. Se considera que la misma debería tener una atención más directa en este tipo de instalación turística, en coordinación con los diferentes actores socioeconómicos locales, para de esta forma lograr una mayor productividad y disminuir las deficiencias existentes que atentan contra la calidad del medio ambiente y el turismo que se desea alcanzar.

La gestión ambiental en la Villa Mirador de Mayabe La Villa Mirador de Mayabe está ubicada en una altura en la localidad del mismo nombre a $12 \mathrm{~km}$ del centro histórico de Holguín desde la cual se obtiene una bella vista de toda la ciudad ubicada en la región oriental de Cuba. En sus inicios era solamente un restaurante pero a través del tiempo ha sido objeto de ampliaciones y remodelaciones 
hasta convertirse en la hermosa Villa que es hoy. Pertenece a la empresa Islazul Holguín, que fue creada como estructura organizativa de la cadena de turismo del propio nombre el 30 de agosto de 1994. Es una estructura empresarial para la administración, promoción y comercialización del producto turístico en el territorio holguinero. A partir de la aplicación del perfeccionamiento empresarial, cuyas bases tienen como objetivo central incrementar al máximo la eficiencia y competitividad de las empresas cubanas, se incluye la dimensión ambiental como elemento fundamental en la gestión, además de esto la instalación se encuentra en un proceso de categorización hotelera según la Norma Cubana NC 127: 2014 "Requisitos para la clasificación de los establecimientos de alojamiento".

En los inicios de la investigación se obtuvo que en la Villa Mirador de Mayabe solamente se limitaban al cumplimiento mínimo de lo establecido en la legislación ambiental vigente. Se adoptaban medidas correctoras a los señalamientos resultantes de inspecciones externas que no suponían cambios significativos, se centraban esencialmente en la proyección de medidas de tratamientos de residuales (fundamentalmente los líquidos), en planes de mejora generales a nivel de instalación que no propiciaban un cambio en los procesos. No se había establecido el cargo del representante de la gestión ambiental ni sus funciones, aunque se ejecutaban programas de ahorro de agua y energía no eran suficientes para su uso eficiente ya que no se enfocaban de forma consciente como mejoramiento del medio ambiente. Hasta ese momento, aunque poseían la estrategia ambiental de la cadena Islazul, no se había adoptado como estrategia general de la instalación, no se implementaban otros mecanismos como la selección de los aspectos legales a cumplir por procesos, las normas y los reconocimientos ambientales, se carecía de un sistema activo que propiciara un vuelco de la situación, hacia un entorno en que prevaleciera el cuidado y respeto ambiental, regido por una cultura de la organización y que contribuyera a la protección permanente del ambiente. La problemática anterior justificó la importancia de desarrollar la presente investigación para dar respuesta al problema científico planteado.

\section{METODOLOGÍA}

Para el procesamiento de los resultados del diagnóstico se utilizaron los métodos estadísticos. El desarrollo de la investigación demandó la utilización de diferentes métodos y técnicas de investigación, estos fueron métodos teóricos y modelos empíricos.

\section{Métodos teóricos}

- El histórico-lógico, de utilidad en la construcción del marco teórico- práctico referencial referido a la gestión ambiental en instalaciones hoteleras enmarcadas en el turismo de naturaleza.

- El análisis-síntesis, para resumir las principales premisas teórico-metodológicas de la relación medio ambiente y turismo, para sintetizar aquellos aspectos positivos y negativos derivados de la consulta a las diferentes biliografías que influyen en la gestión ambiental de la Villa.

- El inductivo-deductivo, utilizado para establecer generalizaciones sobre la base del estudio del proceso de gestión en instalaciones hoteleras enmarcadas en el turismo de naturaleza y para conocer los problemas ambientales actuales que presenta la Villa Mirador de Mayabe.

\section{Métodos empíricos}

- La observación científica, de interés para el estudio del medio ambiente biótico abiótico y socioeconómico e identificar y diagnosticar los problemas ambientales que se presentan en la instalación hotelera.

- La encuesta, la cual fue aplicada a trabajadores y directivos de la entidad para evaluar el nivel de conocimiento sobre el tema.

- La tormenta de ideas, realizada con los especialistas para determinar el estado actual de la gestión ambiental en la Villa y la propuesta de programas de acción.

\section{RESULTADOS}

El aporte fundamental de esta investigación está dado en la aplicación de un procedimiento general que permitió la comprensión y participación de los recursos humanos en el diseño del Sistema de Gestión Ambiental (SGA) y la elaboración de la Estrategia Ambiental (EA) con vistas a sistematizar el desempeño ambiental de la Villa Mirador de Mayabe, una instalación turística que se enmarca dentro de la modalidad de turismo de naturaleza, en conformidad con las normas ambientales vigentes en el sistema de turismo de Cuba. El procedimiento aplicado se aborda a continuación. 


\section{Procedimiento general para la gestión ambiental}

El procedimiento general para la gestión ambiental constituye una herramienta de intervención profesional a utilizar por los recursos humanos de la organización y contempla cada uno de los elementos que propician el desarrollo de acciones desde el diagnóstico hasta la mejora, y presenta los resultados obtenidos en su implementación, con lo que se demuestra el cumplimiento del objetivo general planteado en la investigación. El procedimiento cuenta con cuatro fases y 13 pasos, como se muestra en la Figura 1.

\section{FIGURA 1. Procedimiento general para la gestión am- biental.}

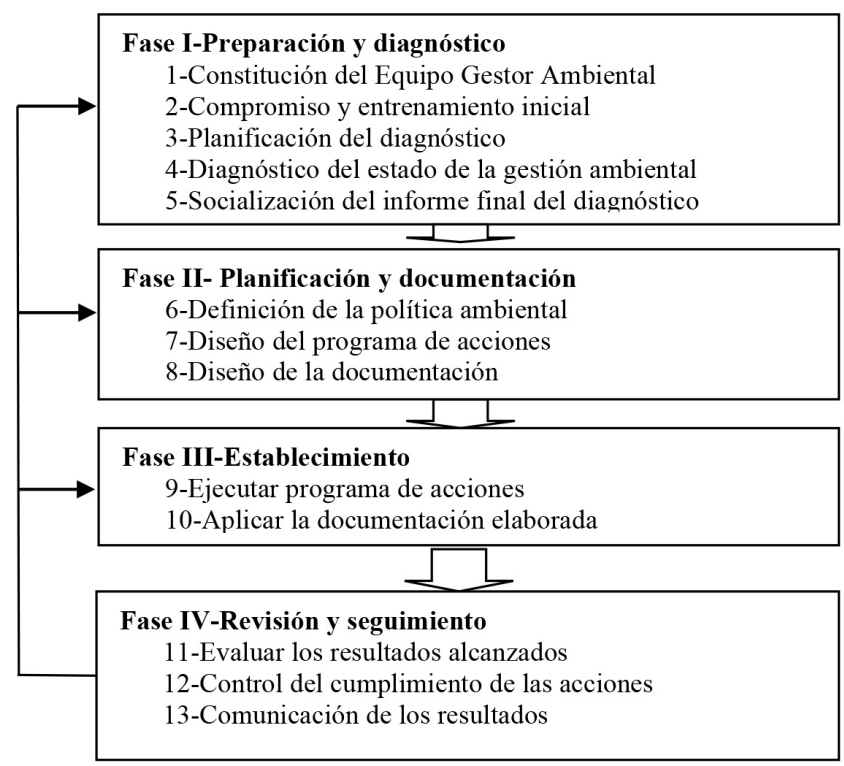

Fuente: Ochoa-Ávila (2014)

Fase I: Preparación y diagnóstico

Esta fase tuvo como objetivo crear las condiciones de partida en la instalación hotelera para identificar los problemas asociados a la gestión ambiental, y determinar las oportunidades de acción para la implantación de la gestión ambiental.

\section{Fase II Planificación y documentación}

El objetivo de la fase fue participar en la planificación de las acciones, para la formulación de la política ambiental, la estrategia y el programa de gestión ambiental, así como organizar el diseño de los procedimientos específicos y demás documentos requeridos para establecer la gestión ambiental en la Villa.

\section{Fase III Establecemiento}

El objetivo de la fase fue ejecutar las acciones de gestión ambiental programadas para contribuir a la transformación ambiental de la instalación hotelera; velar porque fueran aplicados los procedimientos específicos y documentos diseñados para la gestión ambiental; organizar acciones conjuntas de gestión ambiental con las organizaciones del entorno, como vía para la conservación y mejora no sólo del medio ambiente en la instalación hotelera sino también del medio ambiente de la localidad; coordinar y dirigir activamente las acciones de promoción, divulgación y fomento de una cultura sobre gestión ambiental; exigir el cumplimiento del código de conducta ambiental de trabajadores; participar de conjunto con el Consejo de Dirección y trabajadores en la realización de investigaciones relacionadas con la gestión ambiental, así como realizar la presentación de resultados derivados de las mismas ante la Empresa Islazul y la Delegación del Ministerio de Turismo (MINTUR) en Holguín.

\section{Fase IV Revisión y seguimiento}

Esta fase tuvo como objetivo velar por el monitoreo sistemático de los indicadores establecidos para medir y determinar la efectividad del proceso de gestión ambiental; analizar las decisiones tomadas y los resultados alcanzados para que el nuevo ciclo de implantación permita el alcance de las nuevas metas que la instalación hotelera se proponga; comunicar a los trabajadores y demás actores externos y autoridades competentes a través de diferentes canales de comunicación los resultados de la evaluación del proceso de gestión ambiental; promover los resultados de la gestión ambiental en exposiciones y eventos ambientales, y de otra índole donde participen los recursos humanos de la Villa.

\section{Aplicación del procedimiento general para la gestión ambiental}

Se explicitan los resultados de la aplicación del procedimiento general en la Villa Mirador de Mayabe. Se exponen los resultados más significativos que permiten observar el logro de una mejor gestión ambiental, y a su vez comprobar que el procedimiento aplicado permite dar solución al problema científico planteado. 
Fase I: Preparación y diagnóstico

- Paso 1. Constitución del Equipo Gestor Ambiental. Se creó el Equipo Gestor Ambiental (EGA) en la organización en encuentro con los trabajadores. Prevaleció el criterio que estuvieran representados todos los procesos y se designó al Especialista de Calidad como su representante ante el Consejo de Dirección, el que rendirá la información de los resultados de la gestión ambiental. El equipo quedó integrado por los especialistas de Calidad, de Mantenimiento y Aseguramiento, del Área Contable, de Bebidas y Alimentos, de Alojamiento y de Capacitación.

- Paso 2. Compromiso y entrenamiento inicial. Este paso fue de vital importancia para conseguir formar un equipo de personas sensibilizadas y formadas desde el punto de vista ambiental. Fue necesario comprometer y responsabilizar al EGA mediante la impartición de una capacitación por la representante de la gestión ambiental para la familiarización del mismo con los aspectos del diagnóstico a realizar, a través de una tormenta de ideas se identificó el nivel de conocimiento del equipo sobre la gestión ambiental en la instalación hotelera. Este resultado fue utilizado en esta capacitación, con lo cual quedó demostrado que no fue suficiente. Esto motivó que se decidiera realizar una segunda capacitación impartida por especialistas del Centro de Información y Gestión Tecnológica de Holguín, en la que se hizo énfasis en la importancia del diagnóstico, las herramientas a emplear para la realización del mismo, las ventajas de introducir la gestión ambiental en la Villa y en la importancia del compromiso del equipo de realizar con calidad y rigurosidad el diagnóstico.

- Paso 3. Planificación del diagnóstico. En las reuniones realizadas se abordó la importancia del proceso de gestión ambiental en la entidad, se planteó la necesidad de realizar el diagnóstico de los principales aspectos ambientales de cada proceso y se partió del mapa de procesos establecido por el Sistema de Gestión de Calidad, para sentar las bases para el diseño e implantación del procedimiento contextualizado y pertinente en la búsqueda de soluciones a los problemas ambientales a identificar. También fue abordado el procedimiento para el diagnóstico, y se evacuaron todas las dudas del equipo. Fue necesaria la planificación de los recursos de todo tipo para la ejecución de cada aspecto previsto en el diagnóstico, por lo que se conformó una progra- mación detallada de las actividades a desarrollar durante los distintos pasos del diagnóstico, se establecieron los objetivos, las metas, las fechas de cumplimiento, los participantes, recursos necesarios y responsables.

- Paso 4. Diagnóstico del estado de la gestión ambiental. Este paso se llevó a cabo con la participación de todo los integrantes de EGA, el cual arrojó los siguientes resultados: La revisión del presupuesto demostró que tradicionalmente no se había dado inclusión en el plan técnico económico el presupuesto destinado a la actividad de protección ambiental y por medio de la dirección de la empresa Islazul se solicitó a la casa matriz el financiamiento para desarrollar la gestión ambiental en la Villa. Fueron encuestados 17 trabajadores de un total de 73 pertenecientes a todas las áreas de la Villa Mirador de Mayabe para un 22,7\%.

Los resultados reflejados fueron los siguientes: (i) Respecto al conocimiento de los aspectos ambientales asociados a la instalación, el 76,5\% de los encuestados tenía idea de los aspectos ambientales asociados a la instalación. El 76,5\% propone posibles soluciones para contrarrestar los impactos al ambiente. (ii) Aunque no existía una política ambiental declarada el $58,8 \%$ de los trabajadores identificaron que el Consejo de Dirección de la instalación tomaba interés en proteger el medio ambiente. Las acciones identificadas se proyectaban hacia la prohibición de la tala de los árboles, mantenimiento de las áreas verdes, protección de la fauna, higiene en sentido general. (iii) En cuanto a legislaciones, regulaciones, normas ambientales relacionadas con los puestos de trabajo sólo el 5,8\% de los encuestados conocía la existencia de lalegislación. (iv) Respecto a si se realizaban actividades de comunicación, educación, información y sensibilización ambiental en la instalación, el 35,3\% no reconocía que se realizaran este tipo de actividades. El $64,7 \%$ se había informado mediante talleres, por el mural, el código de conducta ambiental, análisis en el consejo de dirección, debates y conversatorios. (v) Respecto a si se clasificaban los desechos sólidos el $47,1 \%$ no conocía si se clasificaban y de las propuestas para mejorar el Plan de Manejo de los desechos refirieron separarlo según su naturaleza, construir un local para los desperdicios y habilitar más cestos. (vi) Respecto a qué indicador de sostenibilidad estaba relacionado con el puesto de trabajo existía confusión en el 100\% de los encuestados. 
Se realizó una revisión documental de los planes de capacitación de años anteriores y se obtuvo que: (i) Dentro del programa de capacitación se valoraba la capacitación de todos los trabajadores en la temática de Seguridad y Salud del Trabajo y no se apreció la planificación de actividades de capacitación sobre la gestión ambiental, aspecto que se tuvo en cuenta para el logro de la eficacia del Sistema de Gestión Ambiental que se diseñó posteriormente.

En el diagnóstico ambiental se detectaron como principales impactos ambientales negativos los siguientes: (i) Vías de acceso a la instalación en deficiente estado técnico lo que provocaba molestia y riesgo en la transportación de las personas hacia la entidad. (ii) La cerca perimetral se mantenía inconclusa lo que hacía latente el riesgo de entrada a personal ajeno a la instalación. (iii) Riesgo de accidentes eléctricos con peligro para la instalación por la existencia de cables eléctricos sin la debida protección y exacerbado por la ausencia de un pararrayos que garantizara la protección de la instalación completa. (iv) Efectos nocivos para la salud de los trabajadores de la cocina y panadería por no funcionar la campana de extracción de gases. (v) Riesgo para la salud humana y el buen funcionamiento de la instalación por el mantenimiento inadecuado del sistema de tratamiento de los residuales líquidos así como la existencia de registros de la conductora del residual sin tapas. (vi) Contaminación probable de aguas subterráneas y al suelo provocado por el vertimiento de residuales líquidos que no eran incorporados por el inapropiado diseño del sistema de tratamiento general de la instalación. (vii) Afectaciones a la higiene y estética de las áreas por la acumulación de residuos sólidos reciclables. (viii) Acumulación de las aguas pluviales en áreas del bloque habitacional con arrastre de material debido a deficiencias en el drenaje. (ix) Arrastres de capa vegetal del suelo en las áreas de pendiente que no poseen césped o vegetación rastrera. $(\mathrm{x})$ Proliferación de vectores y animales domésticos por falta de adecuados Programas Contra Vectores y de Limpieza y Desinfección. (xi) Afectación a la higiene e imagen de la instalación por falta de atención veterinaria a la mascota (burro). (xii) Posibilidad de adquisición de enfermedades profesionales por carencia de equipamiento adecuado en el área de fregado de la vajilla y en el área de servicios del restaurante.

Lo anterior permitió la elaboración de la relación aspecto/ impacto ambiental en los procesos a través de la aplicación de la Matriz de Leopold (Colectivo de autores, 2003). Un resultado del análisis realizado sobre los impactos ambientales negativos en el proceso servicios técnicos se muestra en la tabla 1.

TABLA . Relación de impactos ambientales en el proceso de servicios técnicos.

\begin{tabular}{|c|c|c|c|}
\hline Aspecto asociado & Impacto ambiental & $\begin{array}{c}\text { Carácter } \\
\text { del impacto }\end{array}$ & $\begin{array}{l}\text { Valoración } \\
\text { del impacto }\end{array}$ \\
\hline $\begin{array}{l}\text { Mantenimiento inadecuado } \\
\text { del sistema de tratamiento } \\
\text { de los residuales líquidos }\end{array}$ & $\begin{array}{l}\text { Riesgo para la salud humana } \\
\text { y para el buen funcionamiento } \\
\text { de la instalación y el ecosistema }\end{array}$ & Negativo & Severo \\
\hline $\begin{array}{l}\text { Existencia de cables eléctricos sin la } \\
\text { debida protección y ausencia de } \\
\text { un pararrayos que garantice la } \\
\text { protección de la instalación completa }\end{array}$ & $\begin{array}{l}\text { Riesgo de accidentes eléctricos } \\
\text { con peligro para toda la instalación }\end{array}$ & Negativo & Severo \\
\hline No está completa la cerca perimetral & $\begin{array}{l}\text { Riesgo de entrada de } \\
\text { personal ajeno a la entidad }\end{array}$ & Negativo & Moderado \\
\hline $\begin{array}{l}\text { No existen programas adecuados contra } \\
\text { vectores y de higiene y desinfección }\end{array}$ & $\begin{array}{l}\text { Riesgo de enfermedades de } \\
\text { transmisión alimentarias para } \\
\text { trabajadores y clientes }\end{array}$ & Negativo & Severo \\
\hline Áreas de jardín con pendiente sin césped & Arrastre de la capa vegetal del suelo & Negativo & Moderado \\
\hline
\end{tabular}

Fuente: Elaboración propia 
- Paso 5. Socialización del informe final del diagnóstico. Se procedió a la elaboración del informe del diagnóstico con los datos recogidos. El EGA realizó el análisis de toda la información y la representante de la gestión ambiental redactó la versión final del informe en el que se reflejaron los resultados deficientes, lo que demostró un insuficiente proceso de gestión ambiental en la Villa. El informe fue presentado al Consejo de Dirección el cual tuvo una información precisa de las principales dificultades y de las necesidades para transformar la situación ambiental. Con posterioridad se hizo la presentación del informe por el equipo a los trabajadores, los cuales fueron actualizados sobre los principales problemas detectados en cada proceso y los impactos que su puesto de trabajo genera sobre el medio ambiente, así como las acciones que darían tratamiento a los mismos. Este informe fue comunicado a la dirección de la Empresa Islazul y a la delegación del MINTUR de Holguín.

\section{Fase II: Planificación y documentación}

Una vez concluido el diagnóstico ambiental se determinaron los elementos para la planificación de la gestión ambiental a realizarse por la dirección de instalación hotelera y sus actores, referidos a la formulación de programas y la estrategia de gestión ambiental. Seguidamente se pormenoriza en cada una de sus pasos.

- Paso 6. Definición de la política ambiental. Fue elaborada la política ambiental a seguir según los problemas asociados a los aspectos ambientales de cada proceso identificados en el diagnóstico, y declarada de la manera siguiente: "La Villa El Mirador de Mayabe trabaja para implantar un sistema de Gestión Ambiental basado en la NC ISO 14 001:2004, que conlleve a desarrollar un turismo sostenible". Una vez definida la política se dio a conocer en reunión a los trabajadores, ocasión en la cual se comprometieron a cumplir la misma.

- Paso 7. Diseño del programa de acciones. La dirección de la instalación aprobó las acciones a desarrollar para elevar la gestión ambiental en la Villa "Mirador de Mayabe".
Las fundamentales fueron: (i) Contemplar en el Plan Anual de Capacitación General para la superación sobre gestión ambiental, fundamentalmente en las temáticas de política ambiental cubana, sanciones ambientales, así como otras legislaciones y normativas relacionadas con el medio ambiente, política ambiental de la Villa, principales problemas ambientales generados por la Villa o que afectan a la misma. Con esta medida se dió cumplimiento al artículo 48 de la Ley 81 del Medio Ambiente de 1997. (ii) Desarrollar actividades de educación ambiental de conjunto a los trabajadores y la comunidad circundante, para dar cumplimiento al artículo 47 de la Ley 81 del Medio Ambiente de 1997. (iii) Definir e implantar la política ambiental mediante una estrategia a mediano plazo que garantice su aplicación práctica y el mejor desempeño ambiental de la entidad para cumplir con el artículo 13 incisos a) y g) de la Ley 81 del Medio Ambiente de 1997. (iv) Adoptar medidas técnico organizativas que garanticen la adecuada disposición de los residuales líquidos, para dar cumplimiento al artículo 108 incisos a) y b) de la Ley 81 del Medio Ambiente de 1997. (v) Implantar las medidas técnico-organizativas que garanticen en mantenimiento de la instalación y así cumplir con el artículo 13 de la Ley 81 del Medio Ambiente de 1997. (vi) Realizar la máxima entrega de residuos sólidos reciclables a la Empresa Municipal de Materia Prima para el logro de un mayor aprovechamiento de los mismos y dar cumplimiento al artículo 13 incisos m) de la Ley 81 del Medio Ambiente de 1997.

Se diseñó y se puso en práctica el Programa de Gestión Ambiental según las problemáticas existentes, dentro del cual se conformaron los subprogramas siguientes: (i) Programa de cumplimiento de regulaciones ambientales. (ii) Programa higiénico-sanitario. (iii) Programa de mantenimiento. (iv) Programa energético. (v) Programa de manejo de residuales líquidos y desechos sólidos. (vi) Programa de capacitación y educación ambiental.

- Paso 8. Diseño de la documentación. Fue diseñada la documentación necesaria la cual se redactó e identificó conforme a lo indicado en el procedimiento "Ela- 
boración e identificación de documentos y registros" establecidos en el Sistema de Gestión de Calidad por la Norma Cubana de Calidad de 2008, los documentos elaborados fueron el Manual de Gestión Ambiental, Política Ambiental, Objetivos Metas y Programas Ambientales, Aspectos Ambientales, Recursos, Funciones, Responsabilidad y Autoridad, Diagnóstico Ambiental, Guía para el Diseño del Programa de Gestión Ambiental, Programa Gestión Ambiental, Lista de Chequeo, Indicadores para la sostenibilidad, Código de conducta ambiental para los trabajadores, Código de conducta ambiental para los clientes, Premios ambientales y la Ficha del Proceso de Gestión de Impactos Ambientales. Los procedimientos diseñados fueron: Evaluación de Aspectos Ambientales, Identificación y Acceso a los Requisitos Legales, Preparación y Respuesta ante Emergencias, Cumplimiento de Requisitos Legales, Evaluación del Desempeño Ambiental, Control Operacional Desechos Sólidos, Control Operacional Residuales Líquidos, Control Operacional del Saneamiento de la Piscina, y Conservación de la Flora y la Fauna. En correspondencia con esta documentación se diseñaron los registros para dejar evidencia de la implementación de cada procedimiento.

\section{Fase III: Establecimiento}

- Paso 9. Ejecutar el programa de acciones. Los programas de acciones se ejecutaron conforme a la aprobación de financiamiento para la solución de los problemas priorizados. De conjunto con la empresa Islazul y la inmobiliaria, que es el organismo inversionista del MINTUR se realizan anualmente los análisis sobre el financiamiento necesario para cumplimentar la legislación ambiental y los programas de acciones, los cuales conllevan a la mejora de tecnologías y sistemas. Los planes de financiamiento que se han presentado han sido aprobados por el Grupo Islazul y el MINTUR en el transcurso de los años con lo cual, se ha podido dar solución a los problemas principales que afectaban el buen desempeño ambiental, entre ellos: los relacionados con el sistema de vertimiento de residuales, suministro y red de distribución de energía eléctrica, fue sustituido el equipo de generación eléctrica asistida el cual ocasionaba contaminación al suelo y fueron cam- biadas las pizarras eléctricas obsoletas, en mal estado, desprotegidas y todo el cableado eléctrico que representaba un grave peligro de incendio así como alto consumo de electricidad.

En cumplimiento de compromisos ambientales internacionales y de la propia política del MINTUR se sustituyeron los equipos de climatización y refrigeración por aquellos que no contienen gases contaminantes de la capa de ozono. Esto cumple además con requisitos higiénico-sanitarios debido a que algunos equipos de refrigeración eran obsoletos y se encontraban en condiciones que no cumplían las regulaciones sanitarias. Además se invirtió financiamiento en el completamiento del sistema de protección contra incendio y pararrayos, de gran importancia por las características constructivas de la Villa, con locales construidos con materiales naturales como madera y guano altamente combustibles. Otras de las acciones que se han ejecutado son las reparaciones de las edificaciones, la cubierta de guano de los ranchones, la pintura y carpintería de las habitaciones para solucionar el problema de la entrada de agua de lluvia, así como se ejecutó la remodelación y reparación capital de la piscina, adecuándola a las exigencias higiénicas sanitarias de la Norma Cubana "NC 441 Salud Ambiental. Piscinas. Requisitos Higiénicos Sanitarios y de Seguridad".

Se debe destacar que han sido remodelados espacios para nuevos servicios lo que da cumplimiento a los estándares de la marca Masnatura donde se creó una Eco-cava cerca de la piscina. En un área libre se creó un Tea-garden, ambos ambientados por artistas de las artes plásticas de la ciudad de Holguín. Además, se sustituyó parte del equipamiento para la elaboración de alimentos, se ha modernizó la tecnología para la informatización, se cambió el equipamiento de baños por modelos menos consumidores de agua, se instalaron calentadores solares. Con el presupuesto de mantenimiento se han realizado las reparaciones de mobiliario del lobby y de los restaurantes, de habitaciones así como se ha trabajado en la mejora de las áreas verdes y de los accesos peatonales. En la tabla 2 se muestra un ejemplo de cómo los sistemas fueron mejorados a través del financiamiento recibido de la empresa inmobiliaria en los años 2012 al 2014. 
TABLA 2. Sistemas en los que se invirtió financiamiento por la empresa inmobiliaria del turismo.

\begin{tabular}{ll}
\hline Año & \multicolumn{1}{c}{ Sistema } \\
\hline 2012 & Piscina \\
& Supresores(pararrayos) \\
2013 & Piscina \\
& SADI (Sistema de detección de incendios) \\
2014 & Sistema de bombeo piscina \\
& Sistemas residuales \\
\hline
\end{tabular}

Fuente: Elaboración propia.

Ha sido destacada la contribución del Centro de Capacitación para el Turismo de Holguín en el cumplimiento del plan de capacitación de los trabajadores en diferentes materias que inciden directa o indirectamente en la gestión ambiental, pues en su gran mayoría los programas de formación básica así como los planes de formación de mandos intermedios o de primer nivel tienen incluidos la temática del medio ambiente. El propio EGA se ha encargado de que cada trabajador haya sido instruido en base al Código de conducta ambiental para trabajadores, esto es una acción a desarrollar de forma sistemática. A continuación se muestra el código de conducta para trabajadores.

\section{Código de conducta ambiental de los trabajadores}

- Atiende las quejas y sugerencias de los clientes.

- Cumple con los compromisos individuales de los planes y programas.

- Apaga las luces encendidas cuando no son necesarias.

- Apaga el aire acondicionado cuando no es necesario.

- Manten la hornilla del fogón encendida el tiempo mínimo necesario.

- Manten cualquier tipo de horno encendido el tiempo indispensable.

- Manten la puerta abierta del refrigerador o cámara fría sólo el tiempo necesario.

- Cierra las válvulas de agua mientras no estés utilizándolas.

- Evita utilizar el inodoro como cesto de basura, así ayudaras a ahorrar agua.

- Fotocopia las hojas por las dos caras.

- Utiliza las hojas desechadas que estén escritas por una cara como papel para notas por la otra cara.

- Recoge cualquier desecho que se encuentre en un área no apropiada y colócalo en el depósito adecuado.

- Deposita los cigarrillos apagados o encendidos en los ceniceros y evita tirarlos en las áreas verdes o depósitos de basura.
- Deposita las basuras y desperdicios sólo en los depósitos destinados a ese fin.

- Tira al cesto de basura sólo lo que no pueda ser de utilidad. Piensa antes de tirarlo, en qué puedes utilizarlo.

- Reutiliza las toallas viejas o rotas para la limpieza de pisos y cocina.

- Utiliza la menor cantidad posible de detergentes.

- Utiliza sólo en caso extremo los líquidos decapantes, desgrasantes, etc. y en pequeñas dosis.

- Fumar sólo en las áreas habilitadas para ello.

- Contribuye a que solo se emitan sonidos por debajo de los niveles de ruido permitidos según la Norma Cubana.

- Habla en voz baja. No llames a voces a otra persona.

- Manten una presencia apropiada. Uniforme limpio y aseo personal perfecto.

- Manten una postura erguida y una sonrisa agradable en el desempeño de tus actividades.

- Participa activamente en los matutinos, talleres y cursos sobre medio ambiente y sostenibilidad.

- Lee con detenimiento el Mural Ambiental y aporta para su enriquecimiento.

- Estudia la monografía sobre la caracterización natural, histórica y cultural de la instalación y su comunidad. Brinda esta información de los clientes.

- Cuida las plantas en macetas, jardines y áreas verdes. Evita pisar las áreas verdes, arrancar flores, hojas o ramas y escalar árboles.

- Protege la vida de las mariposas, pajaritos, chipojos, salamandras, lagartijas, jicoteas y otras especies. Ellas son parte de nuestra biodiversidad.

- Respeta las señales de Accesos Prohibidos.

- Solicita para el consumo productos, servicios y tecnologías nacionales, biodegradables y diseñados para el ambiente.

- Brinda especial atención a los clientes discapacitados. 
El Código de Conducta Ambiental de los Trabajadores se ha convertido en un elemento a tener en cuenta en la evaluación del desempeño de los mismos. Las campañas y tareas voluntarias relacionadas con las fechas conmemorativas ambientales constituyen también un eficaz instrumento de formación de conciencia ambiental.

En cuanto al manejo de las relaciones con organizaciones del entorno se realizan actividades en matutinos donde se establecen lazos con escuelas primarias de la localidad circundante y de la ciudad de Holguín, en las que no sólo se hace referencia a la temática del cuidado de la naturaleza sino también a la preservación de las tradiciones culturales y los valores, con las cuales se convoca a concursos en efemérides históricas y ambientales como la celebración del 5 de junio "Día Mundial del Medio Ambiente".

Cada año se divulga con la Emisora de Radio Provincial Radio Angulo de Holguín las actividades por el aniversario de la instalación hotelera donde se resaltan los valores históricos, culturales y ambientales de la misma a través de entrevistas a trabajadores, preguntas a radioyentes y lanzamiento de concursos. Periódicamente se organiza la limpieza del entorno de conjunto con Villa Azúcar, instalación recreativa cercana, y se han establecido contactos con el Consejo Popular para el cuidado del sistema de disposición de residuales líquidos recién reparado.

Los hoteles tienen la particularidad de que sus clientes consumen los servicios en el mismo establecimiento. Este hecho hace imprescindible una buena comunicación con el cliente para que entienda el compromiso ambiental de la instalación y colabore en las actuaciones ambientales dentro de sus posibilidades. Mediante las actividades de animación se ha dado promoción y se ha hecho partícipe a los clientes del conocimiento del Sistema de Gestión Ambiental de la instalación hotelera y de la implementación de su Código de Conducta de Ambiental, una de las formas para invitarle a colaborar activamente en buenas prácticas ambientales es mediante mensajes y pegatinas en las habitaciones. El código de conducta ambiental para los clientes se muestra a continuación.

\section{Código de conducta ambiental para los clientes}

Clients environmental behavior code

- Ayúdanos a proteger el medio ambiente y avanzar hacia un turismo sostenible.

Help us to protect the environment and go towards a sustainable tourism.
- Sugiérenos mejoras en nuestro desempeño ambiental.

Suggest ways to improve our environment performance.

- Protégete cumpliendo con las indicaciones en caso de incendio y catástrofes.

Protect yourself by obeying the instructions concerning what to do in case of fire and/or natural disasters.

- Ayúdanos a reducir el consumo de energía y agua.

Help us to reduce the use of energy and water.

- Cintribuye a mantener las áreas limpias de desechos sólidos.

Help to keep all areas free of litter.

- Deposita los cigarrillos apagados o encendidos en los ceniceros.

Leave cigarette in ashtrays.

- Fuma sólo en las áreas habilitadas para ello.

Smoke only in areas where that are permitted.

- Habla en voz baja.

Speaking low.

- Solicita información sobre la caracterización natural, histórica y cultural de la instalación y su comunidad.

Request information about the natural, historic and cultural features of the Hotel and its local community.

- Evita pisar las áreas verdes, arrancar flores, hojas o ramas y escalar árboles.

Do not walk on the grass; pull up flowers, leaves and/or branches; or climb trees.

- Protege la vida de las mariposas, pajaritos, chipojos, salamandras, lagartijas, jicoteas, peces y otras especies. Estas son parte de nuestra biodiversidad.

Protect the lives of the butterflies, birds, chameleons, salamanders, lizards, turtles, fish and animals of other species. They are part of our biodiversity.

- Permite mantener el cuidado de la vegetación y la fauna. Allows us to take care of plants and animals.

- Respeta las señales de Accesos Prohibidos. Respect "Don't pass" signs.

- Solicita preferentemente productos y servicios nacionales, biodegradables, ecológicos y naturales. Whenever possible, use Cuban biodegradable, ecological, natural products.

- Respeta las costumbres de la comunidad. Respect local customs.

En cuanto a la gestión de compras se han estrechado lazos con productores locales, se ha coordinado y contratado la adquisición de algunos productos siendo significativo, por ejemplo, la compra de vegetales frescos. Más 
del $70 \%$ de los productos alimenticios que se elaboran y venden hoy en la Villa son de producción nacional. Además en la política de compras se prioriza los productos en dosis grandes que generen menos desechos, productos de limpieza biodegradables y se han mantenido contratos con la empresa de materias primas para la recogida de diversos tipos de desechos.

- Paso 10. Aplicar la documentación elaborada. Fue aplicada la documentación diseñada, en lo que jugó un papel primordial el Equipo Gestor Ambiental y el representante del proceso de gestión ambiental. Fueron identificados requisitos legales aplicables al Sistema de Gestión Ambiental, la actualización de estos requisitos se lleva a cabo por varias vías, a través del contrato con la Oficina Territorial de Normalización, con la adquisición de la Gaceta Oficial y mediante el vínculo con los organismos competentes de la provincia Holguín.

Fueron implementados los procedimientos de tratamiento de residuales, en cuanto a los residuos sólidos es notorio el incremento de los volúmenes de entrega a materias primas de algunos renglones, lo cual demuestra el grado de conciencia adquirido y el impacto de la capacitación en los actores internos, aunque ha sido también posible por la creación de condiciones para la clasificación de los mismos en los puestos de trabajo que se generan.

En cuanto al papel de oficina, es reutilizado por ambas caras lo cual redunda en ahorro de dinero para la compra de este renglón, la recogida de residuos reciclables ha propiciado ingresos a la vez que reducen los gastos por disminución de volumen de los desechos no reciclables que son recogidos por Emprestur. La mejora de las condiciones del supiadero de la instalación con enchapes, piso, desagües y clima para los desechos orgánicos contribuyó a la mejor higiene que garantiza la obtención de la licencia sanitaria ya que este era uno de los principales aspectos que lo frenaban, así como el convenio con productores de carne establecidos en el entorno facilitan la recogida de desechos orgánicos para la cría de animales.

Fueron identificados los residuos peligrosos entre los que se encuentran las baterías de los mandos de los televisores y equipos de climatización, los tubos las lámparas fluorescentes, la chatarra electrónica, estos se almacenan conforme a lo establecido en la legislación, los tóner de impresoras se entregan y rellenan a través del servicio de reciclado que ofrece el Grupo Electrónico del Turismo (GET), el aceite y baterías de carros tienen destino final en los talleres de Servisa, empresa de servicio al turismo que tiene plan de manejo de desechos peligrosos, los neumáticos se entregan a la empresa recapadora para su reutilización.

Los procedimientos para el manejo de recursos materiales y naturales como agua, energía y residuos ha contribuido al cumplimiento de las cifras directivas y a la reducción de afectaciones al medio ambiente. Con respecto al plan de consumo de energía eléctrica para su definición se tienen en cuentas los pronósticos de venta extrahotelera y de ocupación por lo que las cifras varían, en el año 2012 se aprecia una notoria disminución del plan debido a que en este periodo se ejecutó la reparación capital de la piscina cuyo sistema de rebombeo no estuvo en funcionamiento así como el servicio del Bar del Burro. En tanto en el 2014 se evidenció un ligero sobreconsumo, lo cual estuvo dado por el sobre cumplimiento en la ocupación prevista.

La implementación de los procedimientos conlleva a (i) Satisfacer las necesidades y expectativas de nuestros clientes, en la prestación de los servicios de alojamiento, gastronomía y recreación, adecuados a sus requerimientos. (ii) Prevenir y mitigar los impactos ambientales negativos más significativos que puedan originarse, así como establecer y evaluar el desempeño ambiental contribuyendo al desarrollo de un turismo sostenible. (iii) Brindar una alimentación sana y segura a nuestros clientes y trabajadores. (iv) Garantizar la salud e integridad física de nuestros trabajadores, clientes y partes interesadas en relación con la prevención y control de los riesgos asociados a los peligros identificados en procesos y actividades. (v) Desarrollar las competencias laborales de un personal motivado e identificado con el mejoramiento continuo. Todo ello para cumplir con la legislación ambiental vigente aplicable a la Villa.

\section{Fase IV: Revisión y seguimiento}

- Paso 11. Evaluar los resultados alcanzados. A través de las auditorías internas al sistema de gestión se 
ha podido llevar a cabo una revisión profunda del sistema, en las que se ha tenido en cuenta las experiencias en la implementación, dificultades para confeccionar registros, las sugerencias de trabajadores y directivos, se verifica el estado de cumplimiento de la legislación y de todas las acciones programadas así como su resultado, en estas auditorías se ha implementado como instrumento el documento Lista de Chequeo Ambiental, la cual cuenta con los siguientes acápites: (i) Política y gestión ambiental. (ii) Administración y política de compras. (iii) Proceso productivo. (iv) Actividad de capacitación ambiental.

De esta forma se han podido detectar las deficiencias que se presentan en el proceso de gestión ambiental y las acciones de mejora propuestas han quedado reflejadas en los registros del procedimiento "Tratamiento de No Conformidades, Acciones Correctivas y Preven- tivas", todo lo cual se lleva a análisis en los consejos de dirección así como se discuten con los trabajadores para lograr la sensibilización permanente y la participación activa de estos en la solución de los problemas.

De forma general fueron incrementados los indicadores y se mejoró la gestión ambiental en la instalación hotelera. La tabla 3 muestra el comportamiento promedio de cada indicador en los últimos cinco años. Ha ocurrido un salto cualitativo fundamentalmente dado por la actitud consciente ante la gestión ambiental de directivos y trabajadores pero no menos importante debido a que el financiamiento destinado y su ejecución han permitido el cumplimiento de acciones primordiales de los programas, todo lo cual ha conllevado a la obtención del aval del cumplimiento legal establecido por el organismo regulador, que corresponde al Ministerio de Ciencia, Tecnología y Medio Ambiente (CITMA).

TABLA 3. Resultados de los indicadores de la gestión ambiental.

\begin{tabular}{lc}
\hline Indicadores & Promedio \\
\hline Cumplimiento de legislación aplicable (CLA) & 93,3 \\
Uso eficaz del financiamiento (UEF) & 100 \\
Tecnología compatible con el medio ambiente (TCA) & 87 \\
Residuos sólidos reciclables (RSR) & 90 \\
Consumo de la energía eléctrica (CEE) & 99 \\
Cumplimiento de las medidas de ahorro y uso racional del recurso agua (CPAURA) & 88 \\
Cumplimiento del plan de capacitación ambiental (CPCA) & 97 \\
\hline
\end{tabular}

Fuente: Elaboración propia.

El indicador residuos sólidos reciclables (RSR) se calculó en base al total de residuales identificados, incluyendo los peligrosos. La gestión es efectiva a partir del 2013 pues más del $80 \%$ de los residuos se reciclan o reutilizan. En el indicador UEF (uso eficaz del financiamiento) es válido destacar que cada año se ha logrado la aprobación de mayores valores para financiar inversiones o reposición de equipos que redundan en la mejora de la gestión ambiental. En el indicador cumplimiento de las medidas de ahorro y uso racional del recurso agua (CPAURA) inciden importantes medidas pendientes como solución de fugas, reutilización de aguas residuales de la piscina para el riego. En el indicador cumplimiento de legislación aplicable (CLA) y tecnología compatible con el medio ambiente (TCA) han evidenciado mayor efectividad a partir las inversiones ejecutadas en el período 2012-2014. En cuanto al indicador consumo de la energía eléctrica (CEE) en 2014 superó el $100 \%$ lo que demuestra ineficacia; sin embargo la empresa Islazul plantea que esta situación está dada debido a que el plan de KW a consumir fue similar al de 2012, año en el que fue menor el consumo aprobado ya que se ejecutó la reparación capital de la piscina.

\section{- Paso 12. Control del cumplimiento de las acciones.} El programa de acciones ambientales es revisado sistemáticamente por el EGA con el objetivo de rectificar alguna deficiencia o no conformidad que surja. De las 87 acciones propuestas al inicio de la investigación se han cumplido 78 para un $89,7 \%$; quedan por realizar fundamentalmente las acciones siguientes: (i) Buscar soluciones para el aprovechamiento de aguas residuales en el riego de jardines. Hay que solicitar a la inmobiliaria la posibilidad de crear un reservorio para 
las aguas de la limpieza de la piscina y que puedan ser monitoreadas y reutilizadas en la jardinería. (ii) Favorecer la anidación de aves mediante la construcción e instalación de nidos artificiales dentro de las áreas de la Villa donde los clientes puedan observar las aves. (iii) Hacer una mini-colección de plantas endémicas en un área que sirva para la educación ambiental de clientes, trabajadores y comunidad. (iv) Identificar el arbolado con sus nombres científicos y vulgares para lograr una apreciación activa de la naturaleza por parte de los clientes. (v) Construir el vivero y dotarlo de las condiciones para hacerlo funcional.

- Paso 13. Comunicación de los resultados. La declaración de la política ambiental expuesta a la vista de clientes y visitantes, proveedores y trabajadores es un instrumento para informar sobre el compromiso ambiental de la Villa. Los resultados de la evaluación del proceso de gestión ambiental fueron comunicados por el Consejo de Dirección a los trabajadores, en los consejos de dirección respectivos al grupo Islazul y a la Delegación territorial del MINTUR de Holguín. El resultado positivo alcanzado en esta instalación hotelera permitió a la empresa Islazul generalizar a todas sus instalaciones este Sistema de Gestión Ambiental. La experiencia del diseño del Sistema de Gestión Ambiental de la Villa Mirador de Mayabe se socializó en el evento científico III Simposio Territorial de Calidad del Turismo de Holguín. Los resultados de un trabajo sostenido han propiciado la obtención de la Licencia Sanitaria y el aval del CITMA, el cual fue ratificado en el año 2014 y se mantiene vigente en el presente.

\section{CONCLUSIONES}

El análisis del marco teórico-práctico demostró que no había sido abordado suficientemente la gestión ambiental en la Villa Mirador de Mayabe. La falta de una estructura adecuada y el desconocimiento de la materia limitaban al cumplimiento de directivas a nivel administrativo, así como el no haber otra instalación con la marca Masnatura que contara con un Sistema de Gestión Ambiental en Cuba.

La implementación del procedimiento general para la gestión ambiental en la Villa Mirador de Mayabe demostró que el dicho procedimiento constituye una herramienta de intervención profesional a utilizar por los propios actores de la organización y contempla cada uno de los elementos que propician el desarrollo de acciones de diagnóstico y mejora, así como la evaluación de los resultados obtenidos en la implementación, con lo que se demuestra el cumplimiento del objetivo general planteado.

El procedimiento general para la gestión ambiental en la Villa Mirador de Mayabe permitió a la instalación hotelera formular e implementar estrategias efectivas para mejorar el medio ambiente y el uso responsable de recursos, adaptándose a los cambios del micro y macro entorno. Además, logró cambios favorables en la generalidad de los indicadores aplicados para su evaluación.

\section{REFERENCIAS}

Atencio, W. (2016). Procedimiento para el diseño e implantación del sistema de gestión ambiental en la empresa inmobiliaria del turismo de Holguín. Tesis de Grado Científico de Máster en Gestión Ambiental. Facultad de Economía, Universidad de Holguín, Cuba.

Carmona-Moreno, E., Magán-Díaz, A. (2008). Estrategia ambiental: definición y tipologías. Disponible en http://feeds.feedburner.com/GestioPolis Consultado el 22 de enero de 15.

Colectivo de autores. (2007). Tabloide de medio ambiente: introducción al conocimiento del medio ambiente. Editorial Academia. 29p

Cruz, E. (2010). Modelo y procedimiento para la gestión ambiental de una zona costera turística Holguín. Diploma para optar al título de Licenciado en Turismo. Universidad de Holguín, Cuba.

CITMA. Estrategia Ambiental Nacional. 2007/2010. Ministerio de Ciencia, Tecnología y Medio Ambiente. Cuba.

CITMA. Estrategia Ambiental Nacional. 2011/2015. Ministerio de Ciencia, Tecnología y Medio Ambiente. Cuba.

Estrategia Ambiental de la Cadena Islazul. (2006). Portador digital. 
Colectivo de autores. (2003). Medio ambiente, impacto y desarrollo. Colección de divulgación científica. Editorial Científico Técnica, p.128-129

González-Abreu, B. (2016). Procedimiento General para la gestión ambiental integral en el hotel Brisas Covarrubias de las Tunas. Tesis para optar al título de Especialista en Gestión Hotelera. Facultad de Ingeniería Industrial y Turismo, Universidad de Holguín.

Ley 81 de Medioambiente. (1997). Ministerio de Ciencia, Tecnología y Medio Ambiente (CITMA).

Manual Operativo de la marca Masnatura. (2012). Grupo hotelero Islazul. Diciembre.

Marrero-Nápoles, L. (2010). Propuesta de estructura organizativa para la gestión ambiental en el polo turístico Guardalavaca. Diploma para optar al título de Licenciado en Turismo. Universidad de Holguín.

Norma Cubana NC 127. 2014. Industria Turística. Requisitos para la clasificación por categorías de los establecimientos de alojamiento turístico.

Norma Cubana NC 441. 2006 Salud ambiental. Piscinas.
Requisitos higiénico sanitarios seguridad.

Norma Cubana NC ISO-14001.2004-Sistema de Gestión Ambiental-Especificaciones con guías para su uso.

Ochoa-Ávila, M. (2014). Tecnología para la gestión ambiental integral en instituciones escolares. Aplicación en Holguín. Tesis de Grado Científico de Doctor en Ciencias Técnicas.

Oficina Nacional de Estadística. (2015). Anuario estadístico provincia de Holguín 2014. Portador digital.

Organización Mundial del Turismo (OMT). (1996). Diary 21 for travel and tourism industry. Towards Environmentally Sustainable Development, Madrid, 175p.

Polanco-Charchabal, P. (2014). Estrategia para el desarrollo del turismo de naturaleza y de aventuras en el destino Holguín. Diploma para optar al título de Licenciado en Turismo. Universidad de Holguín, Cuba.

Salinas-Chávez, E., Salinas, E., Echarri, M. (2008). Geografía turística de Cuba. Apuntes. La Habana. Editorial Feliz Varela. 